\title{
Digital PCR for the Analysis of MYC Copy Number Variation in Lung Cancer
}

\author{
Alexander Brik (D), ${ }^{1}$ Daniel G. Weber, ${ }^{1}$ Swaantje Casjens, ${ }^{1}$ Peter Rozynek, ${ }^{1}$ Swetlana Meier, ${ }^{1}$ \\ Thomas Behrens, ${ }^{1}$ Georgios Stamatis, ${ }^{2}$ Kaid Darwiche, ${ }^{3}$ Dirk Theegarten, ${ }^{4}$ \\ Thomas Brüining, ${ }^{1}$ and Georg Johnen ${ }^{1}$ \\ ${ }^{1}$ Institute for Prevention and Occupational Medicine of the German Social Accident Insurance, Institute of the Ruhr University \\ Bochum (IPA), Bochum 44789, Germany \\ ${ }^{2}$ Department of Thoracic Surgery, University Medicine Essen, Essen 45239, Germany \\ ${ }^{3}$ Department of Interventional Pneumology, University Medicine Essen, Essen 45239, Germany \\ ${ }^{4}$ Department of Pathology, University Medicine Essen, Essen 45239, Germany
}

Correspondence should be addressed to Alexander Brik; bryk@ipa-dguv.de

Received 9 January 2020; Revised 29 June 2020; Accepted 6 September 2020; Published 21 September 2020

Academic Editor: Hubertus Himmerich

Copyright ( 92020 Alexander Brik et al. This is an open access article distributed under the Creative Commons Attribution License, which permits unrestricted use, distribution, and reproduction in any medium, provided the original work is properly cited.

Background. MYC (v-myc avian myelocytomatosis viral oncogene homolog) is one of the most frequently amplified genes in lung tumors. For the analysis of gene copy number variations, dPCR (digital PCR) is an appropriate tool. The aim of our study was the assessment of dPCR for the detection of MYC copy number variations (CNV) in lung tissue considering clinicopathological parameters. Material and Methods. MYC status was analyzed with dPCR as well as qPCR (quantitative PCR) using gDNA (genomic DNA) from tumor and adjacent nontumor tissue samples of lung cancer patients. The performance of $M Y C$ was estimated based on the AUC (area under curve). Results. The results of the MYC amplification correlated significantly between $\mathrm{dPCR}$ and $\mathrm{qPCR}\left(r_{\mathrm{S}}=0.81, P<0.0001\right)$. The MYC copy number revealed by dPCR showed statistically significant differences between tumor and adjacent nontumor tissues. For discrimination, a sensitivity of $43 \%$ and a specificity of $99 \%$ were calculated, representing 55 true-positive and one false-positive tests. No statistically significant differences could be observed for age, sex, and smoking status or the clinicopathological parameters (histological subtype, grade, and stage). Conclusion. The results of the study show that dPCR is an accurate and reliable method for the determination of MYC copy numbers. The application is characterized by high specificity and moderate sensitivity. MYC amplification is a common event in lung cancer patients, and it is indicated that the determination of the $M Y C$ status might be useful in clinical diagnostics.

\section{Introduction}

Lung cancer shows the highest incidence and mortality worldwide with nearly 2.1 million cases and 1.8 million deaths in 2018, respectively [1]. The most common histological type of lung cancer is non-small-cell lung carcinomas (NSCLC) accounting for $85 \%$ of all lung cancer cases [2]. Among other changes, lung cancer is marked by genomic instability resulting in a high frequency of somatic mutations and extensive genomic alterations in individual genomes [3]. Copy number variation (CNV) designates an alteration of genomic DNA characterized by a change of DNA sequence numbers in the normal (diploid) genome. These DNA alterations may affect individual genes, chromosomal regions, or whole chromosomes. It was shown that CNVs are connected with lung cancer as well as several other malignant diseases $[4,5]$. Generally, in cancer, a decrease or increase of DNA copy numbers might affect tumor suppressor genes and oncogenes, respectively.

One of the most frequently amplified genes in lung tumors is v-myc avian myelocytomatosis viral oncogene homolog (MYC) [6]. MYC is located on chromosome $8 \mathrm{q} 24.21$ and codes for a transcription factor playing a role in cell cycle progression, apoptosis, and cellular 
transformation. Using the "The Cancer Genome Atlas" (TCGA) dataset, Schaub et al. showed that MYC is amplified in $21 \%$ of approximately 9,000 samples across 33 different cancers [7]. Particularly, in lung squamous cell carcinoma, MYC is amplified in $37 \%$ of the samples. Thus, MYC alteration might be a promising biomarker candidate for the detection of lung cancer in tissues. Additionally, several studies demonstrated that MYC amplification is associated with poor prognosis in NSCLC and small-cell lung cancer (SCLC) $[8,9]$. However, an increase of MYC gene copies positively affects the response to therapies as seen after treatment of NSCLC by tyrosine kinase inhibitors (TKIs) [10]. Currently, the development of techniques targeting $M Y C$ for cancer therapy is being comprehensively investigated $[11,12]$.

Digital PCR (dPCR) was introduced as a method for precise copy number detection [13]. In general, dPCR is a sensitive, robust, and accurate tool for the detection of low copy targets and rare alleles. In contrast to quantitative PCR (qPCR), dPCR is marked by higher tolerance to enzymeinhibiting substances, higher precision, higher sensitivity, and improved reproducibility [14-17]. Notably, there is no need for a standard curve or calibrator sample. Thus, dPCR might be an accurate tool for the application in clinical diagnostics [18].

The aim of our study was the assessment of dPCR for the detection of MYC CNV in primary lung cancer tissue and adjacent nontumor tissues. Additionally, the MYC copy number status was analyzed considering clinicopathological parameters of the lung cancer patients.

\section{Material and Methods}

2.1. Ethics Statement. All participants of the study provided informed consent. The study was designed according to the rules guarding patient privacy and with the approval from the ethics committee of Faculty of Medicine, Ruhr University Bochum (registration number 4552-12).

2.2. Study Population. Between 03/2013 and 06/2015, 656 subjects with lung cancer, mesothelioma, or other benign diseases of the respiratory system were recruited in the Ruhrlandklinik (Essen, Germany). Within the context of surgical interventions, tissue samples were collected from 241 lung cancer patients.

2.3. Sample Preparation. Removed tissue samples were immediately cooled to $4^{\circ} \mathrm{C}$ and washed with isotonic saline. After pathological evaluation, tissue samples were stored at $-80^{\circ} \mathrm{C}$. For the determination of $M Y C$, tissue samples (tumor and adjacent nontumor tissues without signs of inflammation) from 129 lung cancer patients were used.

2.4. Detection of CNV. Two $40 \mu \mathrm{m}$ sections of frozen tissue were used for isolation of genomic DNA (gDNA). To each tissue sample, $80 \mu \mathrm{L}$ Proteinase K (Qiagen, Hilden, Germany) was added and homogenized for 1 minute with 850$1000 \mathrm{rpm}$ using a Schuett Homgen plus (Schuett Biotec $\mathrm{GmbH}$, Göttingen, Germany). Then, $320 \mu \mathrm{L}$ Buffer ATL (Qiagen) was added and incubated over night at $56^{\circ} \mathrm{C}$ with 1,000 rpm using a ThermoMixer (Eppendorf, Hamburg, Ger- many). Isolation of gDNA was performed using the QIAamp DNA Mini Kit (Qiagen) according to the manufacturer's instructions. Volumes of all reagents were adapted proportionally with increasing template quantity. Quantification of gDNA was performed using a Qubit Fluorometer and Qubit dsDNA BR Assay Kits (Thermo Fisher Scientific, Darmstadt, Germany).

Digestion of $400 \mathrm{ng}$ gDNA was carried out in $16 \mu \mathrm{L}$ Reaction Mix using $10 \mathrm{U}$ EcoRI (BioLabs, Frankfurt am Main, Germany) and 10× EcoRI buffer (BioLabs) for 1 hour at $37^{\circ} \mathrm{C}$ followed for 10 minutes at $65^{\circ} \mathrm{C}$. Preamplification was performed using a MJ Research PTC-200 Thermal Cycler (Bio-Rad Laboratories, Hercules, CA) with $15 \mathrm{ng}$ gDNA as template in $10 \mu \mathrm{L}$ Reaction Mix including $1 \times$ PreAmp Master Mix (Thermo Fisher Scientific), $0.01 \times$ target assay, and $0.01 \times$ reference assay using the following conditions: $95^{\circ} \mathrm{C}$ for 10 minutes, followed by 5 cycles at $95^{\circ} \mathrm{C}$ for 15 seconds, and $60^{\circ} \mathrm{C}$ for 2 minutes.

qPCR was performed using a 7900HT Real-Time PCR System (Thermo Fisher Scientific) with $10 \mathrm{ng}$ gDNA as template as described previously by Mayo et al. [19]. All reactions were performed in triplicates, and nontemplate controls were included in all assays. As a calibrator, $10 \mathrm{ng}$ of human genomic DNA (product code 11691112001; Roche, Mannheim, Germany) was used. CopyCaller v2.0 (Thermo Fisher Scientific) was used for data analysis. The standard deviation of the sample replicates was $<0.13$, the $z$-score $<2.1$, and the $\mathrm{Ct}$ (cycle threshold) of the reference gene was $<32$.

dPCR was carried out using a Dual Flat Block GeneAmp PCR System 9700 (Thermo Fisher Scientific) and QuantStudio 3D Digital PCR 20K Chip Kits v2 (Thermo Fisher Scientific) with $35 \mathrm{ng}$ gDNA as template according to the manufacturer's instructions [20], aiming at a concentration of target and reference between 200 and 2,000 copies/ $\mu \mathrm{L}$. All reactions were performed in duplicates, and nontemplate controls were included. Copy numbers were determined using a QuantStudio 3D Digital PCR Instrument (Thermo Fisher Scientific) and analyzed using the QuantStudio 3D Digital PCR Software version 3.0 (Thermo Fisher Scientific). MYC copy number/nucleus was calculated as $2 \times$ ratio of $M Y C$ copy number to RNase $P$ copy number.

Both qPCR and APCR were performed using the FAMlabeled MYC TaqMan Copy Number Assay (product code Hs02758348_cn; Thermo Fisher Scientific) and the VIClabeled RNase $P$ TaqMan Copy Number Reference Assay (product code 4403326; Thermo Fisher Scientific) as reference. Copy numbers of MYC and RNase $P$ in a sample were measured in parallel using a single chip.

2.5. Statistical Analyses. Statistical analyses were performed using SAS software, version 9.4 (SAS Institute Inc., Cary, NC, USA). The agreement between MYC copy number statuses via different methods was analyzed by Spearman's correlation coefficient and illustrated using Bland-Altman plots. In a Bland-Altman plot, the arithmetic mean of two markers ( $x$ axis) is plotted against the difference between the markers ( $y$ axis). The horizontal line describes the mean of the differences, and the dashed lines describe the 95\% confidence interval (CI) assessed as the mean difference \pm 1.96 standard 
deviation (SD). Dot plots with median and interquartile range (IQR) were used to depict the distribution of single biomarkers. The Mann-Whitney $U$ tests were applied to examine group differences, and the Wilcoxon signed-rank tests were applied to compare two related samples. $P$ values < 0.05 were considered as statistically significant. Receiver operating characteristic (ROC) curves were used to quantify classification performance of the biomarkers. The accuracy of the diagnostic tests was depicted by the area under curve (AUC) and its 95\% CI. Biomarker cut-offs were determined using fixed false positive rates (FPRs), maximum Youden's Index (YI), and set as mean of the biomarker concentration in nontumor tissues plus twofold or threefold SD. The chisquared test was used to compare MYC status and clinicopathological parameters.

\section{Results}

3.1. Study Population. The clinicopathologic parameters of the 129 lung cancer patients are summarized in Table 1. Median age of the patients was 67 years, and $50.7 \%$ of the patients were younger than 67 years. The majority of the patients $(62.0 \%)$ were males. More than half of the participants $(54.3 \%)$ were former smokers, whereas $34.1 \%$ were current smokers and $11.6 \%$ were nonsmokers. Mostly, patients were diagnosed with adenocarcinoma $(51.9 \%)$ or squamous cell carcinoma (23.3\%). The majority of the patients $(83.0 \%)$ were diagnosed with tumor stages $\mathrm{T} 1$ and $\mathrm{T} 2$.

3.2. DNA Treatment Prior to PCR. In order to assess the possible influence of DNA pretreatment, eight sample pairs (consisting of tumor and nontumor gDNA) were either digested, preamplified, or not treated prior to PCR. As shown in Figure 1, the revealed MYC copy numbers of the digested, preamplified, and nontreated gDNA samples were similar and the differences due to the pretreatment were marginal. Notably, in samples with MYC copy numbers $>3$, slightly higher differences between the different pretreatments could be observed. The raw data of the dPCR experiments are presented in Table 1 of the Additional File 1.

3.3. $q P C R$ vs. $d P C R$. For the methodological comparison of the performance of dPCR and qPCR, 116 tumor and nontumor samples from 58 patients were analyzed. Based on the previously obtained results, samples were not treated prior to $\mathrm{dPCR}$ and qPCR. The raw data of the qPCR experiments are presented in Table 2 and of the APCR experiments in Table 3 of the Additional File 1.

As shown in Figure 2(a), results of MYC amplification correlated significantly between $\mathrm{APCR}$ and qPCR for all analyzed groups: tumor and nontumor samples $\left(r_{\mathrm{S}}=0.81, P<\right.$ $0.0001)$, tumor samples $\left(r_{\mathrm{S}}=0.93, P<0.0001\right)$, and nontumor samples $\left(r_{\mathrm{S}}=0.54, P<0.0001\right)$. Additionally, the Bland-Altman plot indicated a good overall agreement between $\mathrm{dPCR}$ and $\mathrm{qPCR}$ in the corresponding samples (Figure 2(b)), i.e., $96.55 \%$ of the measurements fitted within the limit of agreement.
TABle 1: Patients' characteristics and clinicopathological parameters of the study group $(N=129)$.

\begin{tabular}{lcc}
\hline Characteristic & Group & $N(\%)$ \\
\hline $\begin{array}{l}\text { Age (years), median } \\
\text { (range) }\end{array}$ & & $67(39-84)$ \\
Sex & Male & $80(62.0)$ \\
& Female & $49(38.0)$ \\
Smoking status & Never & $15(11.6)$ \\
& Former & $70(54.3)$ \\
& Current & $44(34.1)$ \\
& Adenocarcinoma & $67(51.9)$ \\
Histological subtypes & Squamous cell & $30(23.3)$ \\
& carcinoma & $14(10.9)$ \\
& Carcinoid & $8(6.2)$ \\
& LCNEC & $10(7.8)$ \\
Grade & Other & $11(8.5)$ \\
& G1 & $50(38.8)$ \\
T stage & G2 & $47(36.4)$ \\
& G3 & $8(6.2)$ \\
& G4 & $13(10.1)$ \\
& Unknown & $62(48.1)$ \\
& T1 & $45(34.9)$ \\
& T2 & $3(2.3)$ \\
& Tnknown & $3(2.3)$ \\
\hline
\end{tabular}

${ }^{*}$ LCNEC: large-cell neuroendocrine carcinoma. ${ }^{*}$ Four adenosquamous carcinomas, two non-small-cell lung cancers, one non-small-cell lung cancer (not otherwise specified), two small-cell carcinomas, and one synovial sarcoma. ${ }^{\$}$ Grade 1: well differentiated, low grade; grade 2: moderately differentiated, intermediate grade; grade 3: poorly differentiated, high grade; and grade 4: undifferentiated, high grade.

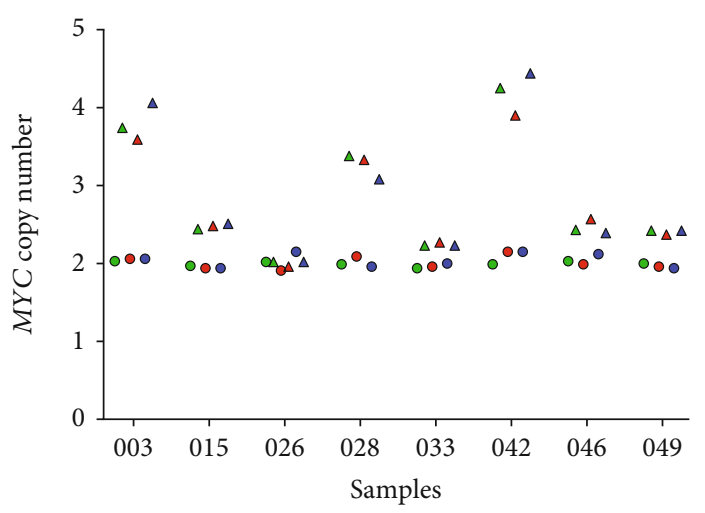

FIgURE 1: MYC copy numbers of samples without and with pretreatment prior to PCR (green: no gDNA pretreatment; red: gDNA digestion with EcoRI; blue: preamplification of gDNA; triangle: tumor samples; circle: nontumor samples).

3.4. Assessing of MYC Copy Number by dPCR. For the assessment of $M Y C$ alteration in lung cancer, $M Y C$ copy numbers were determined in tumor and adjacent nontumor tissue of 129 lung cancer patients using dPCR. The raw data of the 


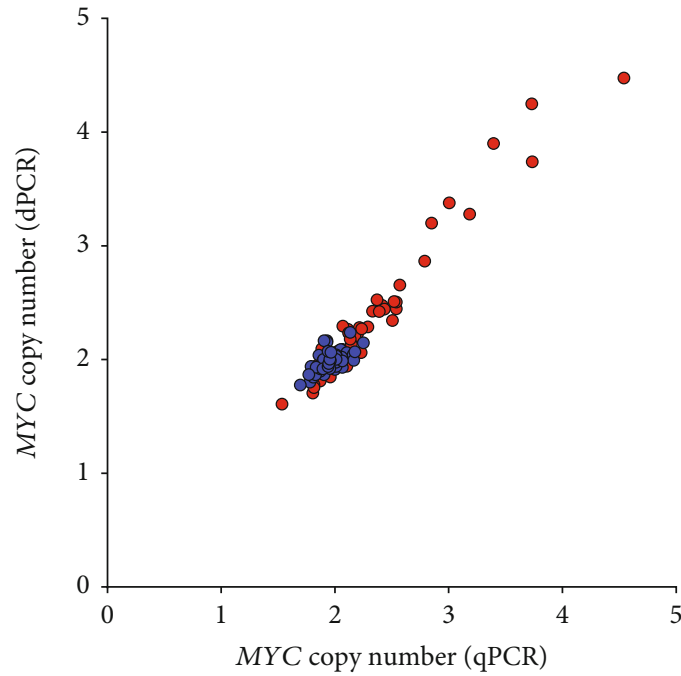

(a)

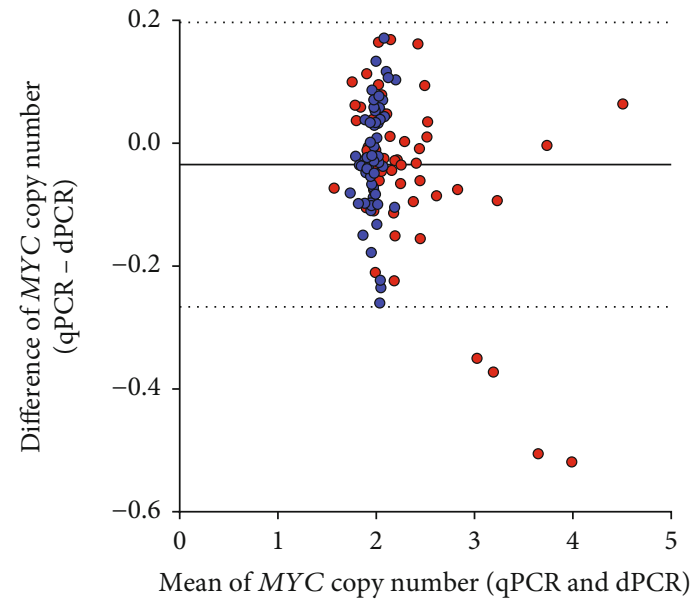

(b)

Figure 2: (a) Correlation of MYC copy numbers in 116 samples (tumor and adjacent nontumor tissue) from 58 lung cancer patients assessed by qPCR and dPCR (red: tumor tissue; blue: adjacent nontumor tissue). (b) Bland-Altman plot of MYC copy numbers assessed by qPCR and dPCR in 116 samples (tumor and adjacent nontumor tissue) from 58 lung cancer patients (red: tumor tissue; blue: adjacent nontumor tissue). The horizontal solid line indicates the mean difference at -0.03 , the horizontal dashed lines indicate the range of $\pm 1.96 \times \mathrm{SD}$ (upper limit $=0.20$; lower limit $=-0.27$ ).

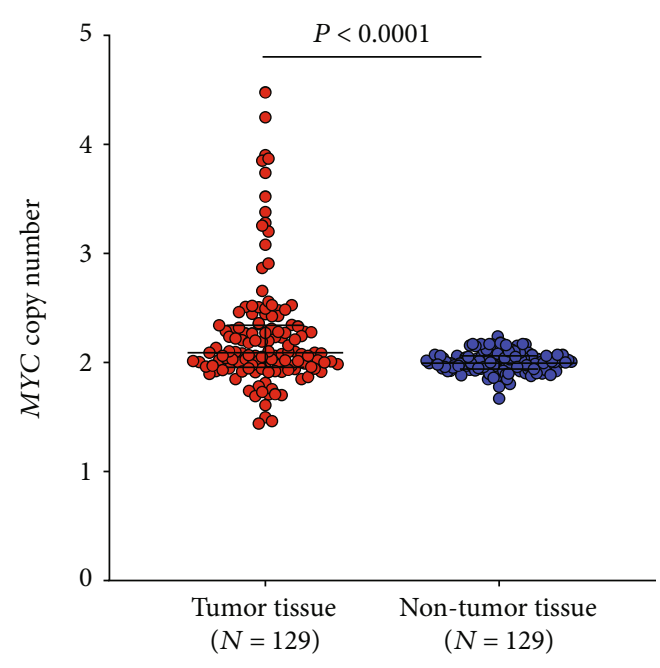

FIgURE 3: Distribution of $M Y C$ copy numbers in tumor and adjacent nontumor tissues from 129 patients. The Mann-Whitney $U$ test was performed to examine group differences. Vertical bars indicate median and interquartile ranges.

dPCR experiments are presented in Table 3 of the Additional File 1. As indicated, no treatment was performed prior to dPCR. The median MYC copy number in tumor samples was 2.09 (IQR: 1.96-2.34) and in nontumor samples 1.99 (IQR: 1.94-2.06). The mean MYC copy number in tumor samples was 2.25 with an SD of 0.53 and in nontumor samples 2.00 with an SD of 0.09. Difference between tumor and nontumor tissues was statistically significant $(P<0.0001)$, (Figure 3).

The ROC analysis revealed an AUC of 0.67 (95\% CI: 0.61-0.74) (Figure 4). As the 95\% CI does not include a value

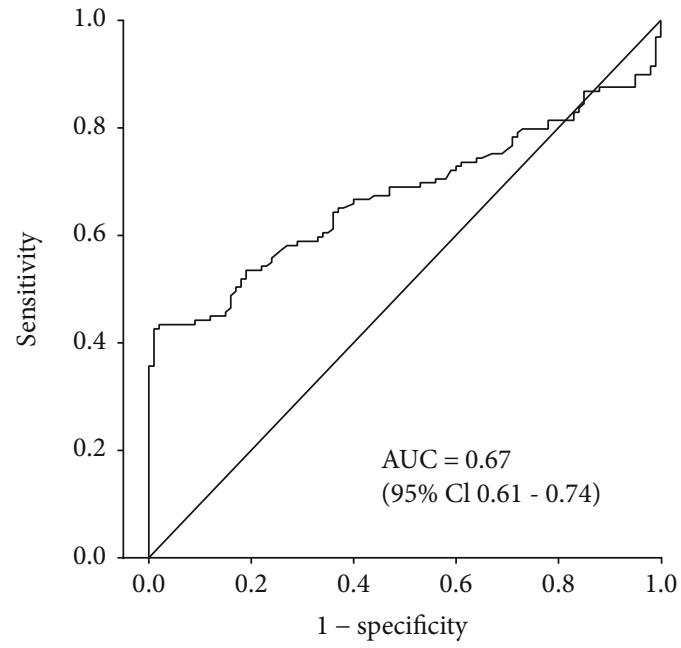

FIgURE 4: Receiver operating characteristic (ROC) analysis for MYC based on tumor and adjacent nontumor tissues from 129 lung cancer patients.

of 0.5 or less, the difference between the AUC and the default AUC of 0.5 is statistically significant.

Application of different cut-offs resulted in slightly different performances (Table 2). Using 95\% specificity (cut-off $=2.17$ ) resulted in $43 \%$ sensitivity, whereas using the mean $+3 \mathrm{SD}$ (cut-off $=2.28$ ) resulted in a lower sensitivity of $30 \%$ but a higher specificity of $100 \%$. The cut-off of 2.19 based on the maximum YI resulted in $43 \%$ sensitivity and 99\% specificity, representing 55 true-positive and one falsepositive test.

MYC copy numbers were analyzed regarding clinicopathological parameters, showing no statistically significant association with age, sex, smoking status, histological 
TABLE 2: Performance of the dPCR-based MYC CNV assay calculated for different cut-offs.

\begin{tabular}{|c|c|c|c|c|c|c|c|}
\hline & Cut-off & $\begin{array}{c}\text { True positive } \\
(N)\end{array}$ & $\begin{array}{l}\text { True negative } \\
(N)\end{array}$ & $\begin{array}{c}\text { False positive } \\
(N)\end{array}$ & $\begin{array}{c}\text { False negative } \\
(N)\end{array}$ & $\begin{array}{c}\text { Sensitivity } \\
(\%)\end{array}$ & $\begin{array}{l}\text { Specificity } \\
\text { (\%) }\end{array}$ \\
\hline 95\% specificity & 2.17 & 56 & 123 & 6 & 73 & 43 & 95 \\
\hline $\begin{array}{l}\text { Maximum Youden's } \\
\text { index }\end{array}$ & 2.19 & 55 & 128 & 1 & 74 & 43 & 99 \\
\hline Mean +2 SD $^{*}$ & 2.19 & 55 & 128 & 1 & 74 & 43 & 99 \\
\hline Mean + 3 SD & 2.28 & 38 & 129 & 0 & 91 & 30 & 100 \\
\hline
\end{tabular}

*SD: standard deviation.

TABLE 3: MYC copy numbers regarding the analyzed clinicopathological parameters $(N=129)$.

\begin{tabular}{|c|c|c|c|c|}
\hline Characteristic & Group & $M Y C \geq 2.19(N=55,42.6 \%)$ & $\begin{array}{c}\text { Tumor } \\
M Y C<2.19(N=74,57.4 \%)\end{array}$ & $P$ value $\left(\mathrm{chi}^{2}\right.$ test $)$ \\
\hline \multirow{2}{*}{ Age, $N(\%)$} & $\leq 67$ years & $31(56.4)$ & $34(45.9)$ & 0.242 \\
\hline & $>67$ years & $24(43.6)$ & $40(54.1)$ & \\
\hline \multirow{2}{*}{ Sex, $N(\%)$} & Male & $34(61.8)$ & $46(62.2)$ & 0.968 \\
\hline & Female & $21(38.2)$ & $28(37.8)$ & \\
\hline \multirow{3}{*}{ Smoking status, $N(\%)$} & Never & $7(12.7)$ & $8(10.8)$ & 0.929 \\
\hline & Former & $29(52.7)$ & $41(55.4)$ & \\
\hline & Current & $19(34.6)$ & $25(33.8)$ & \\
\hline \multirow{5}{*}{ Histological subtypes, $N(\%)$} & Adenocarcinoma & $29(52.7)$ & $38(51.4)$ & $0.338^{*}$ \\
\hline & Squamous cell carcinoma & $12(21.8)$ & $18(24.3)$ & \\
\hline & Carcinoid & $4(7.3)$ & $10(13.5)$ & \\
\hline & LCNEC $^{\#}$ & $6(10.9)$ & $2(2.7)$ & \\
\hline & Other ${ }^{\S}$ & $4(7.3)$ & $6(8.1)$ & \\
\hline \multirow{3}{*}{ Grade, $N(\%)$} & Missing & $5(9.1)$ & $8(10.8)$ & 0.441 \\
\hline & $\mathrm{G} 1+\mathrm{G} 2$ & $23(41.8)$ & $38(51.4)$ & \\
\hline & $\mathrm{G} 3+\mathrm{G} 4$ & $27(49.1)$ & $28(37.8)$ & \\
\hline \multirow{4}{*}{ T stage, $N(\%)$} & Missing & $2(3.6)$ & $1(1.4)$ & $0.092^{*}$ \\
\hline & $\mathrm{T} 1$ & $22(40.0)$ & $40(54.0)$ & \\
\hline & $\mathrm{T} 2$ & $25(45.5)$ & $20(27.0)$ & \\
\hline & $\mathrm{T} 3+\mathrm{T} 4$ & $6(10.9)$ & $13(17.6)$ & \\
\hline
\end{tabular}

${ }^{*}$ Fisher's exact test. ${ }^{*}$ LCNEC: large-cell neuroendocrine carcinoma. ${ }^{5}$ Four adenosquamous carcinomas, two non-small-cell lung cancers, one non-small-cell lung cancer (not otherwise specified), two small-cell carcinomas, and one synovial sarcoma.

subtype, grade, and stage (Table 3). In general, most samples within the different groups were $M Y C$ negative, i.e., the $M C Y$ copy number was $<2.19$. Notably, increased numbers of samples from patients with large-cell neuroendocrine carcinomas (LCNEC) and patients with stage T2 (six out of eight and 25 out of 45, respectively) were $M Y C$ positive (copy number $\geq 2.19$ ), but differences were statistically not significant.

\section{Discussion}

MYC is a classical oncogene, and its amplification is a common event in lung cancer. In this study, we detected an increase of $M Y C$ copy numbers in $43 \%$ of the analyzed lung cancer samples. Published MYC alterations varied widely between $11 \%$ and $88 \%[8,21]$. The large range in published $M Y C$ alterations might be based on several factors, e.g., dif- ferent study groups, different methods, and different cutoffs applied.

The performance of the MYC CNV assay utilizing dPCR is marked by a moderate sensitivity of $43 \%$ and a very high specificity of $99 \%$, resulting in only one false positive test. In comparison, Flacco et al. revealed $61 \%$ sensitivity and $60 \%$ specificity using FISH (fluorescence in situ hybridization) for the detection of MYC copy numbers in a cohort of 113 NSCLC patients [22]. Applying a higher specificity of nearly $99 \%$ resulted in reduced sensitivity of approximately $20 \%$. Interestingly, nearly all LCNEC were MYC positive, whereas barely half of the patients with other histological subtypes were MYC positive. Additionally, higher levels of $M Y C$ were observed in $\mathrm{T} 2$ tumors in contrast to $\mathrm{T} 1$ and $\mathrm{T} 3+\mathrm{T} 4$ tumors. The cause for the increased $M Y C$ amplification in LCNEC and in T2 tumors remains unknown. To our knowledge, no significant differences were indicated for these groups 
in other studies $[8,10,22]$. However, the number of samples in the corresponding groups was low. Thus, these observations need to be verified in larger studies.

Common methods for the assessment of MYC CNV in tissues were FISH, CISH (chromogenic in situ hybridization) $[23,24]$, CGH (comparative genome hybridization) $[25,26]$, and $\mathrm{qPCR}[8,27]$. However, in contrast to dPCR, FISH, $\mathrm{CSIH}$, and $\mathrm{CGH}$ are expensive and time-consuming methods, depending on subjective assessment $[15,28]$. In comparison to $\mathrm{qPCR}, \mathrm{dPCR}$ is marked by higher accuracy and reproducibility [24] and there is no need for a standard curve or calibrator sample within an analysis. Thus, dPCR might be a user-friendly method for the application in clinical diagnostics.

dPCR for the determination of MYC copy numbers has been performed only in a small number of studies for different tumor entities [28]. To our knowledge, analysis of $M Y C$ $\mathrm{CNV}$ in lung cancer using $\mathrm{dPCR}$ has not been described before. We show that the determination of MYC copy numbers using dPCR and qPCR revealed comparable results. Similarly, Yong et al. indicated a high correlation of dPCR and qPCR analyzing AMY2A (amylase alpha 2A) and $A M Y 1$ (amylase 1) in whole blood from obese and nonobese subjects [29]. Additional, Shoda et al. demonstrated a correlation between $\mathrm{dPCR}$ and qPCR analyzing HER2 (human epidermal growth factor receptor 2) in plasma of gastric cancer patients [30]. Thus, dPCR appears to be a reliable tool for the analysis of copy numbers [18].

A pretreatment of the gDNA prior to dPCR was suggested in order to improve the performance [31, 32]. For the analysis of MYC copy numbers, we used untreated as well as pretreated (digested and preamplified) gDNA. Basically, no differences were revealed for MYC copy numbers between treated and nontreated gDNA. However, differences could be observed for samples with $M Y C$ copy numbers $>3$. As differences occur only for copy numbers above, the cut-off of 2.19 and the quantitation of the exact copy number might not be necessary; at least for the analysis of the MYC status using the described method, a pretreatment of the gDNA is not required. In agreement with our results, several studies showed that pretreatment did not improve the performance of copy number detection [28, 33-35]. This might be based on already fragmented gDNA or low gDNA concentration $(<3 \mathrm{ng} / \mu \mathrm{L})$ in the samples [32].

It was suggested that lung cancer is marked by increased $M Y C$ copy numbers showing more aggressive phenotypes. In addition, it was indicated that MYC amplification was correlated with poor prognosis in small-cell lung carcinomas [9], NSCLC [22], and adenocarcinomas [8]. Unfortunately, no information was available regarding the survival time of the patients in this study.

Nevertheless, we confirmed that MYC amplification is a frequent event in lung cancer patients and may have high potential in clinical diagnostics for patient selection regarding personalized therapies. As Cappuzzo et al. reported that patients with amplified $M Y C$ were more sensitive to treatments with TKIs [10], the precise and fast assessment of the MYC status using dPCR might improve and speed up the decision trees in clinical routine.
A limitation of this study is the small number of samples in the analyzed subgroups, e.g., LCNEC as well as stages T3 and T4, because higher numbers would enable more meaningful analyses. Unfortunately, no information regarding the survival of the patients analyzed in this work was available, although the use of $M Y C$ as a prognostic marker might be interesting.

\section{Conclusion}

In conclusion, we demonstrated that $\mathrm{APCR}$ is an accurate and reliable method for the assessment of the MYC status in tissues, showing that $M Y C$ amplification is a common event in lung cancer patients. The performance of the MYC copy number determination using a fixed high specificity of $99 \%$ is marked by a moderate sensitivity of $43 \%$. Assessment of MYC status by dPCR might be useful in clinical diagnostics as a prognostic marker and for selecting an appropriate therapy. A future goal would be the development of a bloodbased dPCR assay to determine the MYC status minimal invasively.

\section{Data Availability}

The raw data supporting the conclusions of this article are included as Additional File 1.

\section{Conflicts of Interest}

The authors declare that there is no conflict of interest regarding the publication of this paper.

\section{Authors' Contributions}

$\mathrm{AB}$ conceived the study, participated in its design and coordination, performed the experiments, and drafted the manuscript. DGW conceived of the study, participated in study design and coordination, and drafted the manuscript. SC performed the statistical analyses and helped to draft the manuscript. PR and SM participated in study design and helped to draft the manuscript. TBe coordinated field work, participated in study design, and helped to draft the manuscript. GS, KD, DT, and TBr participated in study design and coordination. GJ conceived the study, participated in study design and coordination, and helped to draft the manuscript. All authors have read, commented, and approved the manuscript.

\section{Acknowledgments}

The authors gratefully acknowledge Bettina Dumont, Simone Naumann, and Katja Szafranski for the excellent technical assistance. We thank Antje Müller and Sigrid Semke for the documentation of samples, registration of study data, and sample biobanking.

\section{Supplementary Materials}

Additional File 1 Table 1: levels of RNaseP (copies/ $\mu \mathrm{L}$ ) and MYC (copies/ $\mu \mathrm{L})$ by dPCR. Table 2: levels of RNaseP 
(Ct) and MYC (Ct) by qPCR. Table 3: levels of RNaseP (copies $/ \mu \mathrm{L}$ ) and MYC (copies $/ \mu \mathrm{L})$ by dPCR. (Supplementary Materials)

\section{References}

[1] F. Bray, J. Ferlay, I. Soerjomataram, R. L. Siegel, L. A. Torre, and A. Jemal, "Global cancer statistics 2018: GLOBOCAN estimates of incidence and mortality worldwide for 36 cancers in 185 countries," CA: a Cancer Journal for Clinicians, vol. 68, no. 6, pp. 394-424, 2018.

[2] H. S. Kim, T. Mitsudomi, R. A. Soo, and B. C. Cho, "Personalized therapy on the horizon for squamous cell carcinoma of the lung," Lung cancer (Amsterdam, Netherlands), vol. 80, no. 3, pp. 249-255, 2013.

[3] V. Jabs, K. Edlund, H. König et al., "Integrative analysis of genome-wide gene copy number changes and gene expression in non-small cell lung cancer," PLoS One, vol. 12, no. 11, article e0187246, 2017.

[4] A. M. Bowcock, "Invited review DNA copy number changes as diagnostic tools for lung cancer," Thorax, vol. 69, no. 5, pp. 495-496, 2014.

[5] D. G. Albertson, "Gene amplification in cancer," Trends in Genetics : TIG, vol. 22, no. 8, pp. 447-455, 2006.

[6] W. W. Lockwood, R. Chari, B. P. Coe et al., "DNA amplification is a ubiquitous mechanism of oncogene activation in lung and other cancers," Oncogene, vol. 27, no. 33, pp. 4615-4624, 2008.

[7] F. X. Schaub, V. Dhankani, A. C. Berger et al., "Pan-cancer alterations of the MYC oncogene and its proximal network across the cancer genome atlas," Cell Systems, vol. 6, no. 3, pp. 282-300.e2, 2018.

[8] R. Iwakawa, T. Kohno, M. Kato et al., "MYC amplification as a prognostic marker of early-stage lung adenocarcinoma identified by whole genome copy number analysis," Clinical Cancer Research : an official journal of the American Association for Cancer Research, vol. 17, no. 6, pp. 1481-1489, 2011.

[9] R. d. C. S. Alves, R. T. Meurer, and A. V. Roehe, "MYC amplification is associated with poor survival in small cell lung cancer: a chromogenic in situ hybridization study," Journal of Cancer Research and Clinical Oncology, vol. 140, no. 12, pp. 2021-2025, 2014.

[10] F. Cappuzzo, M. Varella-Garcia, E. Rossi et al., "MYC and EIF3H coamplification significantly improve response and survival of non-small cell lung cancer patients (NSCLC) treated with gefitinib," Journal of Thoracic Oncology: Official Publication of the International Association for the Study of Lung Cancer, vol. 4, no. 4, pp. 472-478, 2009.

[11] L. Soucek and G. I. Evan, "The ups and downs of Myc biology," Current Opinion in Genetics \& Development, vol. 20, no. 1, pp. 91-95, 2010.

[12] H. Chen, H. Liu, and G. Qing, "Targeting oncogenic Myc as a strategy for cancer treatment," Signal Transduction and Targeted Therapy, vol. 3, no. 1, p. 5, 2018.

[13] S. Weaver, S. Dube, A. Mir et al., "Taking qPCR to a higher level: analysis of $\mathrm{CNV}$ reveals the power of high throughput qPCR to enhance quantitative resolution," Methods, vol. 50, no. 4, pp. 271-276, 2010.

[14] I. Hudecova, "Digital PCR analysis of circulating nucleic acids," Clinical Biochemistry, vol. 48, no. 15, pp. 948-956, 2015.
[15] B. J. Hindson, K. D. Ness, D. A. Masquelier et al., "Highthroughput droplet digital PCR system for absolute quantitation of DNA copy number," Analytical Chemistry, vol. 83, no. 22, pp. 8604-8610, 2011.

[16] A. S. Whale, J. F. Huggett, S. Cowen et al., "Comparison of microfluidic digital PCR and conventional quantitative PCR for measuring copy number variation," Nucleic Acids Research, vol. 40, no. 11, pp. e82-e82, 2012.

[17] R. Sanders, J. F. Huggett, C. A. Bushell, S. Cowen, D. J. Scott, and C. A. Foy, "Evaluation of digital PCR for absolute DNA quantification," Analytical Chemistry, vol. 83, no. 17, pp. 6474-6484, 2011.

[18] J. F. Huggett, S. Cowen, and C. A. Foy, "Considerations for digital PCR as an accurate molecular diagnostic tool," Clinical Chemistry, vol. 61, no. 1, pp. 79-88, 2015.

[19] P. Mayo, T. Hartshorne, K. Li, C. McMunn-Gibson, K. Spencer, and N. Schnetz-Boutaud, "CNV analysis using TaqMan copy number assays," Current Protocols in Human Genetics, vol. 67, no. 1, 2010.

[20] Thermo Fisher Scientific, Quant Studio 3D Digital PCR System. Quick Reference (Pub. no. MAN0008159 Rev. D), Thermo Fisher Scientific, 2015.

[21] H. Kubokura, T. Tenjin, H. Akiyama et al., "Relations of the cmyc gene and chromosome 8 in non-small cell lung cancer: analysis by fluorescence in situ hybridization," Annals of Thoracic and Cardiovascular Surgery, vol. 7, no. 4, pp. 197-203, 2001.

[22] A. Flacco, V. Ludovini, F. Bianconi et al., "MYC and human telomerase gene (TERC) copy number gain in early-stage non-small cell lung cancer," American Journal of Clinical Oncology, vol. 38, no. 2, pp. 152-158, 2015.

[23] A. N. Seo, J. M. Yang, H. Kim et al., "Clinicopathologic and prognostic significance of c-MYC copy number gain in lung adenocarcinomas," British Journal of Cancer, vol. 110, no. 11, pp. 2688-2699, 2014.

[24] D. H. Hwang, H. Sun, S. J. Rodig, J. L. Hornick, and L. M. Sholl, "Myc protein expression correlates with MYC amplification in small-cell lung carcinoma," Histopathology, vol. 67, no. 1, pp. 81-89, 2015.

[25] G. Johnen, M. Krismann, M. Jaworska, and K. M. Müller, "CGH-Befunde bei neuroendokrinen Tumoren der Lunge," Der Pathologe, vol. 24, no. 4, pp. 303-307, 2003.

[26] W. Li and M. Olivier, "Current analysis platforms and methods for detecting copy number variation," Physiological Genomics, vol. 45, no. 1, pp. 1-16, 2013.

[27] N. Pfarr, R. Penzel, F. Klauschen et al., "Copy number changes of clinically actionable genes in melanoma, non-small cell lung cancer and colorectal cancer-a survey across 822 routine diagnostic cases," Genes, Chromosomes \& Cancer, vol. 55, no. 11, pp. 821-833, 2016.

[28] K. S. Lee, S. K. Nam, S. H. Seo et al., "Digital polymerase chain reaction for detecting c-MYC copy number gain in tissue and cell-free plasma samples of colorectal cancer patients," Scientific Reports, vol. 9, no. 1, article 1611, 2019.

[29] R. Y. Y. Yong, S.'. A. B. Mustaffa, P. S. Wasan et al., "Complex copy number variation of AMY1 does not associate with obesity in two east Asian cohorts," Human Mutation, vol. 37, no. 7, pp. 669-678, 2016.

[30] K. Shoda, D. Ichikawa, Y. Fujita et al., "Monitoring the HER2 copy number status in circulating tumor DNA by droplet digital PCR in patients with gastric cancer," Gastric Cancer: 
official journal of the International Gastric Cancer Association and the Japanese Gastric Cancer Association, vol. 20, no. 1, pp. 126-135, 2017.

[31] J. Qin, R. C. Jones, and R. Ramakrishnan, "Studying copy number variations using a nanofluidic platform," Nucleic Acids Research, vol. 36, no. 18, article e116, 2008.

[32] Bio-Rad, Droplet Digital PCR: Applications Guide, Bulletin_ 6407, 2014.

[33] A. S. Whale, S. Cowen, C. A. Foy, and J. F. Huggett, "Methods for applying accurate digital PCR analysis on low copy DNA samples," PloS One, vol. 8, no. 3, article e58177, 2013.

[34] A. Alanio, A. Sturny-Leclère, M. Benabou, N. Guigue, and S. Bretagne, "Variation in copy number of the $28 \mathrm{~S}$ rDNA of Aspergillus fumigatus measured by droplet digital PCR and analog quantitative real-time PCR," Journal of Microbiological Methods, vol. 127, pp. 160-163, 2016.

[35] M. C. Kline, E. L. Romsos, and D. L. Duewer, "Evaluating digital PCR for the quantification of human genomic DNA: accessible amplifiable targets," Analytical Chemistry, vol. 88, no. 4, pp. 2132-2139, 2016. 